\title{
Immunomodulating, Neuroprotective and Regenerative Modali- ties in Multiple Sclerosis Management
}

\author{
Mike KS Chan', Michele Zocchi², Dina Tulina', Anam K Kour ${ }^{3}$, Lau Cher Rene ${ }^{4}$, Roni Moya ${ }^{5}$ and Michelle \\ WB Fah ${ }^{6}$
}

${ }^{1}$ Stellar Biomolecular Research, Germany

${ }^{2}$ Department of Plastic, Reconstructive and Regenerative Surgery, University of Padua, Italy

${ }^{3}$ Normah Medical Specialist Centre, Malaysia

${ }^{4}$ European Wellness Academy, Malaysia

${ }^{5}$ Cooperativa de Ensino Superior Politécnico e Universitário, Portugal

${ }^{6}$ European Wellness Academy, Germany

*Corresponding author: Dr. Dina Tulina, MD, Stellar Biomolecular Research, Klosterstrasse 205, Edenkoben, Germany

\begin{abstract}
The incidence of Multiple sclerosis disease is rapidly increasing worldwide, for instance, across Asia, the utmost growth was confirmed in Japan from 1,000 in 1980 to more than 20,000 people in 2019 [1]. The phenomenon of "Westernization" in Asian countries which includes a specific choice of food and lifestyle was related to the higher prevalence of MS in Asia's population which almost supports new findings on the great influence of gut and its microbiota in people worldwide. The current conventional therapy helps to prolong the periods between attacks and relapses, but in fact, does not slow down the disease progression and impact on physical activities which eventually leads to disabilities. The development of a new approach and data is highly required with the opportunity to avoid immunity suppression. This review article shares information on the complex integrative approach of the management of MS cases with the application of immune-modulating peptides, mitochondria derived neural peptides, precursor stem cells, active specific immunotherapy therapy and diet. The results showed a regeneration effect, stable prolonged remission, a reversal of symptoms, reduction of side effects, high efficacy of reduction of MS lesions as well as safety of combination (ongoing and integrative) approach.
\end{abstract}

\section{Introduction}

Multiple sclerosis (MS) is a continuing disabling disease of the central nervous system that is prevalent in the northern hemisphere [2]. A worldwide number of people who live with MS are more than 2.3 million [3], a debilitating condition triggered by an autoimmune response that causes inflammation damage to nerve cells in the central nervous system and currently incurable. MS is highly often diagnosed in females than males about 2 to 3 times more common in relapsing-remitting MS [4]. This phenomenon also shared with several other autoimmune diseases, like rheumatoid arthritis. MS most commonly affects people between the ages of 20 and 50 years and also might be presented in childhood or late middle age [5]. Researchers have found that MS affects different age groups differently. For instance, people who are diagnosed when they are 50 years or older typically have a more progressive disease course. MS is determined as a chronic inflammatory disease of the brain and spinal cord with focal lymphocytic infiltration, which eventually harms myelin sheaths and axons [6]. Primarily, inflammation is temporal, and remyelination happens but is not permanent, and as such, the early stages of MS are presented by incidences of neurological dysfunction, which is next followed by recovery [7]. Anyway, after some time, the pathological changes become inevitable and constant by proliferation microglial activation related to comprehensive and chronic neurodegeneration which clinically appear as a progressive accumulation of disability [8].

Citation: Chan MKS, Zocchi M, Tulina D, Kour AK, Rene LC, et al. (2020) Immunomodulating, Neuroprotective and Regenerative Modalities in Multiple Sclerosis Management. Int J Neurodegener Dis 3:017. doi.org/10.23937/2643-4539/1710017

Accepted: September 08, 2020; Published: September 10, 2020

Copyright: (C) 2020 Chan MKS, et al. This is an open-access article distributed under the terms of the Creative Commons Attribution License, which permits unrestricted use, distribution, and reproduction in any medium, provided the original author and source are credited. 
The pathogenesis of MS is not well known, and there is a multitude of factors that may cause the onset of this disease. Genetic factors may play a major role [9], whereas other studies have indicated a correlation between pathogenic infections and the development of the disease [10]. Several genetic variations are shared within different autoimmune diseases suggesting several common pathogeneses [11]. Currently, the most widely accepted hypothesis is that MS is an autoimmune disease that affects genetically predisposed individuals afflicted with an environmental pathogen [12]. Research results of the last decades indicated that the etiology of most autoimmune diseases includes environmental features that overlap the genetically efficient profiles $[13,14]$. The location of affected nerve fibers plays a major role in the clinical appearance of MS signs and symptoms and might differ significantly during the course of the disease. It might affect legs and trunk or any limb with numbness or weakness, dizziness, spasticity, the vision disappearance (partial or full), tingling or pain in parts of your body; electric-shock sensations during the neck movements (Lhermitte's sign), unclear speech, fatigue and balance problems, lack of coordination or unsteady gait, also can be problems with bowel and bladder function.

The inflammation during MS attacks destroys a fatty substance that protects the nerve fibers, called myelin, which causes damage to nerve cells, nerve fibers itself and the cells that produce myelin, called oligodendrocytes [15]. MS is a highly heterogeneous disease with very diverse pathological and clinical manifestations. The early stages of MS represent and characterized by prevailing of perivascular inflammatory infiltrates typically in the brain, optic nerve, and spinal cord and consist of lymphocytes (CD4+ and CD8+ T cells as well as B cells), monocytes, and macrophages and eventually generate plaques [16]. The end-stage of MS differentiated by demyelination, astrogliosis, and neuronal as well as axonal degeneration $[17,18]$. It was confirmed that crucial part in all process lead by dendritic cells that invade CNS that present antigen a further activate autoreactive T cells $[19,20]$. Furthermore, innate and adaptive immunity cells, like CD8+ T cells directly harm axons the secretion of granzyme B and perforin [21] as well as macrophages might injure CNS by producing toxic molecules like nitric oxide, oxygen radicals and proinflammatory cytokines [22]. The commonly accepted pathologic criteria of demyelinating diseases are 1) Demolition of the myelin sheaths of nerve fibers with comparative sparing of the different components of nervous tissue, i.e., of axons, nerve cells, and supporting structures, as reflected by a relative lack of Wallerian or secondary degeneration of fiber tracts; 2 ) Infiltration of inflammatory cells in a perivascular and particularly paravenous distribution; 3) A spread of lesions that is mainly in white matter, either in multiple small disseminated foci or in larger foci spreading from one or more centers.
The diagnosis made after MRI evoked potential test and spinal fluid examination and to confirm a diagnosis of MS, a person should present signs of disease in different parts of the nervous system and at least two separate flare-ups. MRI examination demonstrates inflammatory lesions and axonal loss. Evoked electrophysiological potentials show the interference of conduction in previously myelinated pathways [23]. The analysis of cerebrospinal fluid confirms intrathecal synthesis of oligoclonal antibody. In individuals with complex genetic-risk profiles, the environmental factors can trigger the clinic and evaluation of multiple sclerosis $[24,25]$. Activation of microglia as a consequence of chronic inflammation may also activate neurotoxic pathways (e.g. production of pro-inflammatory cytokines, proteolytic enzymes, reactive oxygen and nitrogen species) that induce damage neurons and further progress neurodegeneration [26]. During the different MS stages, inflammation and neurodegeneration apparently coexist, trending more to an inflammatory process in early MS, and toward neurodegeneration with disease progression [27]. Microglia consists of extremely specialized cells, that might induce neurotoxic pathways or exert stimulation of neuroprotection, reduction of inflammation, and promotion of repair which mechanisms predominate will depend on the stage and context of any given lesion [28].

\section{Methods}

The most approved drugs and medicine using in MS patients demonstrated the decrease of the amount of new episodes but in fact, did not reverse occurred deficits and as such have a doubtful impact on the long-term cumulative disability and MS progression. The prevention of long-term effects continues to be an issue as well as medicine for the treatment of progressive MS stages which also have limitations including their efficacy. Hence, new, improved disease management is essential to effectively treat MS [29]. The most of MS treatments are long term firstly suppressing the immune system; however, such immune-inhibitors relationship increase risks for infectious diseases and cancer [30]. The first stem cells transplantation was conducted in 1997 in a chronic myelogenous leukemia patient with MS, which demonstrated significant improvements in MS brain lesions [31]. It was shown that stem cells in patients with active MS, decrease progression in about $70 \%$ of patients, reduce relapses considerably and inhibits inflammatory MRI activity [32]. The method includes observation of MS cases during and after the complex and integrative approach on disease management during the period of five years on average. Symptomatic therapeutic effects of most paths are considered proven. However, it would be advantageous to combine therapeutic modalities including immune modulators, neural peptides, organ specific cell therapy and diet to observe reliably stable results. 


\section{Results}

The therapy intention with disease-modifying agents in patients with MS includes shortening of the duration of acute exacerbations, decreasing their frequency, and providing symptomatic relief [5]. No curative, FDA-approved therapies for MS are currently available. Symptomatic treatments are aimed at maintaining function and improving quality of life [33]. At the present time, MS disease has no cure; however, some medications are available that can alleviate symptoms and slow disease progression, decrease the amount of relapses or attacks, and to decrease their severity when they do occur. In order to achieve such results, the medications usually prescribed in the form of injections. High doses of steroids are used to treat relapses but have tremendous side effects. Multiple numbers of other medications and lifestyle interventions may be used to help manage symptoms such as bladder problems, tiredness, pain, depression, anxiety, and stress, but there doesn't impact the actual disease directly. Various rehabilitation programs, including physical and occupational therapy, are an important part of supporting people living with MS.

\section{Immune modulating peptides}

Inflammation is invariably present in almost all lesion types and disease stages of MS [34]. Nearly the majority of current actual MS treatment does not control specific immune cells which eventually suppress the general immune response and cause a lot of negative side effects opportunistic pathogens. Therefore the immune-modulating agents are required in such state to regulate myelin-reactive immune response and defend against other infections and foreign invaders. The nano-size peptides with short chains of 20 or less amino acids become the valuable specific modulators of protein-mediated signaling of the immune system. The effect of nano-sized immune-modulating peptides based on restoring immune balance without causing global immune suppression [35]. The technology uses specific peptides, which induce an antigen-specific expansion of regulatory $T$ cells - the critical mediators of immune tolerance. These cells can inhibit autoantigen-specific helper T cells, which secrete proinflammatory cytokines and are responsible for immune reactions in autoimmune diseases such as MS. The results revealed the deactivation of existing MS lesions and stable remission on chronic conditions. It was also found a strong movement towards improvement in the Multiple Sclerosis Functional Composite (MSFC) score, which is a reliable indicator of improvement in MS course and disability.

\section{Mitochondria derived neural peptides}

Neural Mito Organelles (MO) Formula combines 10 kDa nano-size peptides with 45-65 kDa. Mitochondria in cells are a fundamental structure for energy and destructions within mitochondrial complexes of oxidative phosphorylation definitely direct to cell death. Disruptions or damages of this type of organelle are also indicated in some neurodegenerative diseases, for instance, Parkinson's disease and Huntington's disease [36,37]. The energy expenditures of a neuron for conducting an impulse in conditions of demyelination increase; however, cells cannot always be provided with energy enough due to inflammatory damage to the respiratory complexes.

Neural MO reduces neuron death, save the integrity of myelin and increase the survival of myelin-producing cells. Improve motor function, which becomes significantly better in subjects treated with the MO peptides compared to controls. The Neural MO contributed substantially to remyelination in demyelinated neurons. In particular, the Neural MO peptides do not perform as suppressors to immune cells as well as do not deteriorate the neuron transmission in the brain, which described as a usual negative effect of treatment that affects the glutamate system, however, ameliorated survival of myelin-producing cells.

\section{Organ specific cell therapy}

Precursor stem cells (PSCs) are cell with the capacity to differentiate into only one cell type that is why individual prescription for every patient is required by the medical or scientific board. PSCs showed the restorative capacity on self-tolerance, provide immunomodulation and neuroprotection, and promote regeneration in patients with chronic diseases such as multiple sclerosis. Studies showed that PSC with oligodendrocyte precursor cells effectively regenerate nerve cells, protect myelin from damage, replicate myelin-producing cells and as such, ameliorate and recover neurological functioning. MS brain is amenable for repair, and PSC therapy has an advantage in comparison to other strategies to enhance endogenous remyelination as neural PSC will substitute damaged oligodendrocytes and myelin as well as efficiently weaken the autoimmune process in a local, nonsystemic way to protect brain cells from onward injury and contribute the essential capacity of the brain for recovery [38].

These fundamental immunomodulatory and neurotrophic properties are shared by precursor stem cells of different sources. PSC can target both affected white matter tracts and the perivascular niche where the trafficking of immune cells occurs. Stem cells showed itself as an effective therapy in regeneration medicine and a novel approach to prevail on the limitations of presently existing MS management. Stem cell use is now available all over the world to treat MS [39]. Neural precursor stem cells provide a source of remyelinating cells with the ability to structurally repair the central nervous system [40]. Another achievement is the replacement and resentment of the entire immune system. PSC is a potentially effective and safe treatment for MS. Neural precursor stem cells has a unique capacity of remye- 
lination. Mesenchymal precursor stem cells (MPSCs) transform into neurons and endothelial cells after induction, and they also secrete factors with various functions such as neurogenesis, inhibition of apoptosis (programmed cell death), neuronal and glial cell survival, expansion of axonal and myelin repair processes, development and protection of nervous tissue and integration, and improvement of local stem cells. MPSCs have the ability to transdifferentiate into (become) neuron-like cells, so they also have the same ability to deliver cell substitutes to the injured central nervous system. PCS is a reasonable and advanced way of repairing inflamed and impaired tissue.

The cytokines of MPSCs mediate immune modulating features to defend tissue and contribute in the repair which has been well demonstrated both in vitro and in animal and human models placing them as the main capable therapy for clinical states characterized by inflammation or tissue damage inclusive MS [41]. MPSCs found to be able to proliferate $T$ cells, reduce Th1 proinflammatory responses with perfect tolerability and safety. It was reported that a syngeneic MPSCs improve clinical outcome by diminishing demyelination and leucocytes infiltration in the CNS [42].

\section{Active Specific Immunotherapy (ASI) therapy}

The idea of anti-idiotypic antibodies as regulatory agents were primarily stated by Niels Jerne in the Network Hypothesis more than 40 years ago [43], where was expressed that this exceptional feature of antibodies both to recognize an antigen and be recognized by other antibodies as an antigen establish a balanced network which functions as a monitor of the humoral arm of the immune system $[44,45]$. Anti-idiotypic antibodies are suggested to manage the homeostasis of the adaptive humoral immune responses by neutralizing idiotypic antibodies and controlling idiotypic antibody secretion $[46,47]$. Anti-idiotypic immunity can be a potent and very specific way of inducing immunosuppression and generating tolerance. Because the natural occurrence of anti-idiotypic antibodies to idiotypes associated with autoantibodies has already been suggested in a few instances, the possibility that regulation of autoreactivity may be under idiotypic control is not unlikely [48]. This hypothesis is maintained by the availability of anti-idiotypic antibodies in healthy individuals and in patients in remission from autoimmune diseases, and by the lack of anti-idiotypic antibodies during active disease [49]. Thus, it is admissible to think that the production of autoantibodies may be preferred by lack of down-regulation of autoreactive clones at their emergence by quantitatively insufficient or functionally inefficient anti-idiotypes or anti-idiotype-driven regulatory mechanisms. Conversely, it is understandable that it might also be possible that anti-idiotypes cause direct potentiation of responsiveness to self and/or autoimmune processes. Elucidating the role of network regulation on the reactivity to autoantigens is important not only for clarifying the physiological role of idiotype-anti-idiotype interactions in the maintenance of immunological tolerance vis-à-vis the components of the body constituents but also for designing new strategies of treatment for autoimmune diseases such as MS. ASI become a fundamental strategy itself and within complex approach in the management of MS. The observation showed that ASI reduced disease progression, decreased the number of relapses as well as new attacks, and to minimize their severity if such an event happened.

The basis of ASI in the management of MS is the generation of an individualized 'vaccine' that targets the idiotypic antibodies that are present in the body. It uses the patient's own unique individual idiotypic antibody to generate an exact anti-idiotypic antibody. The introduction of this anti-idiotypic antibodies leads to the formation of the ligand complexes, that would initiate an adaptive cell-mediated immune chain reaction against the patient's 'own antibody-antigens'. Anti-idiotypic immunity is a potent and very specific way of inducing immunosuppression and generating immune tolerance.

The ASI therapy modulates the localized dysfunctional immune balance in the CNS of the MS afflicted individuals.

\section{Diet}

Research showed that environmental factors such as occupation, climate, chronic stress, smoking, as well as diet, have a serious impact on the development of autoimmune diseases, including MS [50]. It was confirmed that the Western diet, which includes high salt, high fat, high protein, and high sugar leads to growing cases of some common diseases [51,52]. The changes in consumption and food combination showed a correlated effect on the immune system that ultimately affects homeostasis [53] and also on bacterial colonization of the gastrointestinal tract [54] as the gut microbiota is closely bound with the immune system and strongly engaged in immune regulatory processes [55]. In Inflammatory bowel disease were demonstrated changes and different reduction in the microbiome also the same confirmed in other autoimmune diseases which not directly associated with the gastrointestinal tract $[51,56]$. Nowadays, the gut and its microbiota are in high consideration in many fields and health research. The human gut microbiome is assumed to include up to $10^{14}$ bacteria from around 500-1000 species which support the body in homeostasis [57]. The first acquaintance to the microbiome happens in birth time and breastfeeding with continuing impact on the colonization of the newborn's own gut microbiome [58-60]. It was confirmed that gut dysbiosis, changes in intestinal immunity [61] might activate the pro-inflammatory Th17 cells [62,63], as well as play a role in MS due to the induction of relapsing-remitting autoimmune disease [64]. Also, the latest report in the gut-brain-axis system may onwards 
promote a potential bond between gut microbiota and autoimmunity [65]. The low-calorie diets, which include fruits, fish and vegetables, were included in complex of MS management which was proved to downregulate proinflammatory molecules and recover or support healthy symbiotic gut microbiota [66]. The diet was composed of plant-derived nutrients which associated with an anti-inflammatory potential $[67,68]$. The diet was advised for life-long with crucifers like broccoli, the vegetables which inhibit the secretion of proinflammatory cytokines [69] as well as products containing retinoic acid [68] which play a role in stimulating and maintaining regulatory T cells $[70,71]$. Most negative impact caused by diets rich in fat and salt studied as potential risk factors for MS and was avoided in MS management.

The gut is made up of a complex microbial ecosystem. The gut houses almost $80 \%$ of the cells to support the immune system. The mucosal lining in the gut as the largest lymphoid tissue in the body is often known as "our forgotten organ". These microbes play an important role in the immune system, digestive system, metabolic system, cardiovascular system and central nervous system. Gut microbiome become imbalance when there is disruption among healthy and unhealthy microbes. Dysbiosis is found to be associated with psoriatic arthritis, rheumatoid arthritis, and other autoimmune diseases. Healthy microbes also produce Vitamin K2 in the gut. Vitamin K2 plays a key role in our body [72]. It is a critical nutrient for optimal cellular function. Vitamin K2, especially menaquinone-4 (MK-4), is responsible for processes at the genetic and metabolic level. It is important in mitochondrial function, ATP production, myelin sheath production, immune system modulator, activates anti-oxidants and detoxification pathways $[73,74]$. Vitamin $\mathrm{K} 2$ is anti-inflammatory factors and shown to reduce the severity of relapse in MS [75]. Research has found that vitamin $\mathrm{K} 2$ level is lower in MS patient. There are many clinical studies on the role of vitamin K2 in MS shows the beneficial effects of vitamin K2 on the nervous system [76].

\section{Conclusions}

Broad strokes management of MS has hitherto been systemic immune suppression, the use of disease-modifying drugs (DMDs) therapies in the relapsing forms of MS, the monitoring of progression of the disease and providing associated medical support and physical therapies/aids for the neuro-disabilities. The focus of the treatments has been in relieving symptoms, controlling acute exacerbations, shortening the duration of acute relapses, reducing the frequency of relapses, and in the longer term, slowing down the disease progressions. The complex MS management by immune-modulating and neural peptides along with PSC, ASI and lifestyle modifications including a change of diet showed it effectiveness in frequency reduction of relapse period, shorter patient's recovery duration, and the effect was observed in long-term with neuroprotective changes and enhanced quality of life in mild and moderate MS stages. No serious adverse events were reported. The mechanism of action of PSC includes immunomodulation, neuroprotection and neuroregeneration. The use of PSC that reduce MRI parameters is a new and emerging research focus to develop new, improved treatments for MS.

MS pathogenesis is highly complicated, comprising various affiliates of the immune system, and so far still stay not well known. Current drugs for MS therapy are almost non-specific, creating symptoms as they surfaced, resulting in a weaker immune system and causing inhibition of immune reactions against infections. Consequently, the improvement of current and development of new MS management approach is in high demand with the possibility to escape general suppression of immunity. In this article were present an integrative approach of applying a combination of immune-modulating peptides, neural peptides, Precursor Stem Cells (PSCs), Active Specific Immunotherapy (ASI) therapy coupled with lifestyle modifications for the management of MS. The modality of such therapy adds a new dimension, geared to further enhancing in the management and sustaining the acquired long-term results of MS. The potential reduction of the side effect was demonstrated on common drugs treatment as well as improvement on efficacy and safety of whole provided MS therapy (traditional and integrative). The synergy of both modalities not only provides better quality of patient's life but also aims to reverse the symptoms in the sequence order of occurrence with a goal towards remission ultimately.

\section{Acknowledgements}

Declared none.

\section{Conflict of Interest}

The author confirms that this article content has no conflict of interest.

\section{References}

1. Yamamura T (2017) ECTRIMS Online Library. 202391: 48.

2. Simpson S Jr, Blizzard L, Otahal P, Van der Mei I, Taylor B (2011) Latitude is significantly associated with the prevalence of multiple sclerosis: a meta-analysis. J Neurol Neurosurg Psychiatry 82: 1132-1141.

3. Browne $P$, Chandraratna D, Angood C, Tremlett H, Baker C (2014) Atlas of multiple sclerosis 2013: A growing global problem with widespread inequity. Neurology 83: 10221024.

4. Compston A, Coles A (2002) Multiple sclerosis. Lancet 359: 1221-1231.

5. Cree BAC (2007) Multiple sclerosis. In: Brust JCM, Current diagnosis and treatment in neurology. Lange Medical Books/McGraw-Hill Medical, New York. 
6. Lassmann $H$ (2010) What drives disease in multiple sclerosis: Inflammation or neurodegeneration? Clin Exp Neuroimmunol.

7. Janet E Joy, Richard B Johnson (2001) Multiple sclerosis: Current status and strategies for the future. Review.

8. Correale J, Marrodan M, Ysrraelit MC (2019) Mechanisms of neurodegeneration and axonal dysfunction in progressive multiple sclerosis. Biomedicines 7: 14.

9. Oksenberg JR, Baranzini SE, Sawcer S, Hauser SL (2008) The genetics of multiple sclerosis: SNPs to pathways to pathogenesis. Nat Rev Genet 9: 516-526.

10. (2018) International Multiple Sclerosis Genetics Consortium. Low frequency and rare coding variation contributes to multiple sclerosis risk. bioRxiv.

11. Cotsapas C, Hafler DA (2013) Immune-mediated disease genetics: the shared basis of pathogenesis. Trends in Immunology 34: 22-26.

12. Jörg S, Grohme DA, Erzler M, Binsfeld M, Haghikia A (2016) Environmental factors in autoimmune diseases and their role in multiple sclerosis. Cell Mol Life Sci 73: 4611-4622.

13. Manzel A, Muller DN, Hafler DA, Erdman SE, Linker RA (2014) Role of "Western diet" in inflammatory autoimmune diseases. Curr Allergy Asthma Rep 14: 404.

14. Bogdanos DP, Smyk DS, Rigopoulou El, Mytilinaiou MG, Heneghan MA (2012) Twin studies in autoimmune disease: Genetics, gender and environment. J Autoimmun 38: J156-J169.

15. National Multiple Sclerosis Society (NMSS).

16. Popescu BF, Pirko I, Lucchinetti CF (2013) Pathology of multiple sclerosis: where do we stand? Continuum (Minneap Minn) 19: 901-921.

17. Hohlfeld R, Dornmair K, Meinl E, Wekerle H (2016) The search for the target antigens of multiple sclerosis, part 2: CD8+ T cells, B cells, and antibodies in the focus of reverse-translational research. Lancet Neurol 15: 317-331.

18. Hohlfeld R, Dornmair K, Meinl E, Wekerle H (2016) The search for the target antigens of multiple sclerosis, part 1: Autoreactive CD4+ T lymphocytes as pathogenic effectors and therapeutic targets. Lancet Neurol 15: 198-209.

19. Lande R, Gafa V, Serafini B, Giacomini E, Visconti A (2008) Plasmacytoid dendritic cells in multiple sclerosis: Intracerebral recruitment and impaired maturation in response to interferon-beta. J Neuropathol Exp Neurol 67: 388-401.

20. Serafini B, Rosicarelli B, Magliozzi R, Stigliano E, Capello E (2006) Dendritic cells in multiple sclerosis lesions: Maturation stage, myelin uptake, and interaction with proliferating T cells. J Neuropathol Exp Neurol 65: 124-141.

21. Irani DN (2005) Immunological mechanisms in multiple sclerosis. Clin Appl Immunol Rev 5: 257-269.

22. Zindler E, Zipp F (2010) Neuronal injury in chronic CNS inflammation. Best Practice \& Research Clinical Anaesthesiol 24: 551-562.

23. Compston A, Coles A (2008) Multiple sclerosis. Lancet 372: 1502-1517.

24. Gourraud PA, Harbo HF, Hauser SL, Baranzini SE (2012) The genetics of multiple sclerosis: An up-to-date review. Immunol Rev 248: 87-103.

25. Waubant E, Lucas R, Mowry E, Graves J, Olsson T (2019) Environmental and genetic risk factors for MS: An integrated review. Ann Clin Transl Neurol 6: 1905-1922.
26. Correale $\mathrm{J}$ (2014) The role of microglial activation in disease progression. Mult Scler 20: 1288-1295.

27. Thompson AJ, Baranzini SE, Geurts J, Bernadd Hemmer, Olga Ciccarelli (2018) Multiple sclerosis. Lancet 391: 16221636.

28. Czeh M, Gressens P, Kaindl, AM (2011) The yin and yang of microglia. Dev Neurosci 33: 199-201.

29. Dargahi N, Katsara M, Tselios T, Androutsou ME, de Courten M, et al. (2017) Multiple Sclerosis: Immunopathology and treatment update. Brain Sci 7: 78.

30. Inglese M, Petracca M (2015) Therapeutic strategies in multiple sclerosis: A focus on neuroprotection and repair and relevance to schizophrenia. Schizophr Res 161: 94101.

31. McAllister LD, Beatty PG, Rose J (1997) Allogeneic bone marrow transplant for chronic myelogenous leukemia in a patient with multiple sclerosis. Bone Marrow Transplant 19: 395-397.

32. Mancardi G, Saccardi R (2008) Autologous haematopoietic stem-cell transplantation in multiple sclerosis. Lancet Neurol 7: 626-636.

33. Brunton LL (2005) Immunomodulators. In: Lazo JS, Parker KL, Goodman \& Gilman's the pharmacological basis of

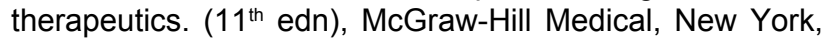
1424-1427.

34. Frischer JM, Bramow S, Dal-Bianco A, Lucchinetti CF, Rauschka $\mathrm{H}$, et al. (2009) The relation between inflammation and neurodegeneration in multiple sclerosis brains. Brain 132: 1175-1189.

35. Tulina D, Chan MKS, Michele WBF, Pan SY (2019) Transfer Factors or Dialyzable leukocyte Extracts as Immunomodulating Peptides: A Conceptual Review on Broad Spectrum of Therapeutic Areas, Immunologic and Clinical Responses, Trends and Perspectives. Int J Immunol Immunother 6: 039.

36. Harald Hampel, Gordon Wilcock, Sandrine Andrieu, Paul Aisen, Kaj Blennow, et al. (2011) Biomarkers for alzheimer's disease therapeutic trials. Prog Neurobiol 95: 579593.

37. Jin YN, Johnson GVW (2010) The interrelationship between mitochondrial dysfunction and transcriptional dysregulation in Huntington disease. Journal of Bioenergetics and Biomembranes 42: 199-205.

38. Ben-Hur T (2011) Cell therapy for multiple sclerosis. Neurotherapeutics 8: 625-642.

39. Shroff G (2018) A review on stem cell therapy for multiple sclerosis: special focus on human embryonic stem cells. Stem Cells Cloning 11: 1-11.

40. Chandran S, Hunt D, Joannides A, Zhao C, Compston A, et al. (2008) Myelin repair: The role of stem and precursor cells in multiple sclerosis. Philos Trans R Soc Lond B Biol Sci 363: 171-183.

41. Lianhua Bai, Donald P Lennon, Valerie Eaton, Kari Maier, Arnold I Caplan, et al. (2009) Human bone marrow-derived mesenchymal stem cells induce Th2-polarized immune response and promote endogenous repair in animal models of multiple sclerosis. Glia 57: 1192-1203.

42. Emanuela Zappia, Simona Casazza, Enrico Pedemonte, Federica Benvenuto, Ivan Bonanni, et al. (2005) Mesenchymal stem cells ameliorate experimental autoimmune encephalomyelitis inducing T-cell anergy. Blood 106: 17551761. 
43. Jerne NK (1974) Towards a network theory of the immune system. Ann Immunol (Paris) 125: 373-389.

44. Jerne NK (1974) Clonal selection in a lymphocyte network. Soc Gen Physiol Ser 29: 39-48.

45. Jerne NK (1973) The immune system. Sci Am 229: 52-60.

46. Kim BS (1982) Mechanisms of idiotype suppression: role of anti-idiotype antibody. Surv Immunol Res 1: 126-132.

47. Rodkey LS (1980) Autoregulation of immune responses via idiotype network interactions. Microbiol Rev 44: 631-659.

48. Maurizio Zanetti (1983) Anti-Idiotypic antibodies and autoantibodies. Medical Biology Institute, La Jolla, California. Annals of the New York Academy of Sciences.

49. Hampe CS (2012) Protective role of anti-idiotypic antibodies in autoimmunity--lessons for type 1 diabetes. Autoimmunity 45: 320-331.

50. Marrie RA (2004) Environmental risk factors in multiple sclerosis aetiology. Lancet Neurol 3: 709-718.

51. Thorburn AN, Macia L, Mackay CR (2014) Diet, metabolites, and "western-lifestyle" inflammatory diseases. Immunity 40: 833-842.

52. Odegaard AO, Koh WP, Yuan JM, Gross MD, Pereira MA (2012) Western-style fast food intake and cardiometabolic risk in an Eastern country. Circulation 126: 182-188.

53. Riccio P, Rossano R (2015) Nutrition facts in multiple sclerosis. ASN Neuro 7.

54. Conlon MA, Bird AR (2015) The impact of diet and lifestyle on gut microbiota and human health. Nutrients 7: 17-44.

55. Kuhn KA, Stappenbeck TS (2013) Peripheral education of the immune system by the colonic microbiota. Semin Immunol 25: 364-369.

56. Salonen A, de Vos WM (2014) Impact of diet on human intestinal microbiota and health. Annu Rev Food Sci Technol 5: 239-262.

57. Neish AS (2009) Microbes in gastrointestinal health and disease. Gastroenterology 136: 65-80.

58. Fanaro S, Chierici R, Guerrini P, Vigi V (2003) Intestinal microflora in early infancy: Composition and development. Acta Paediatr Suppl 91: 48-55.

59. Marcobal A, Sonnenburg JL (2012) Human milk oligosaccharide consumption by intestinal microbiota. Clin Microbiol Infect 18: 12-15.

60. Penders J, Thijs C, Vink C, Stelma FF, Snijders B, et al. (2006) Factors influencing the composition of the intestinal microbiota in early infancy. Pediatrics 118: 511-521.

61. Chassaing B, Gewirtz AT (2014) Gut microbiota, low-grade inflammation, and metabolic syndrome. Toxicol Pathol 42: 49-53.

62. Cani PD, Bibiloni R, Knauf C, Waget A, Neyrinck AM, et al. (2008) Changes in gut microbiota control metabolic endo- toxemia-induced inflammation in high-fat diet-induced obesity and diabetes in mice. Diabetes 57: 1470-1481.

63. Veldhoen M, Hirota K, Westendorf AM, Buer J, Dumoutier L, et al. (2008) The aryl hydrocarbon receptor links TH17-cell-mediated autoimmunity to environmental toxins. Nature 453: 106-109.

64. Berer K, Mues M, Koutrolos M, Rasbi ZA, Boziki M, et al. (2011) Commensal microbiota and myelin autoantigen cooperate to trigger autoimmune demyelination. Nature 479: 538-541.

65. Mayer EA (2011) Gut feelings: The emerging biology of gutbrain communication. Nat Rev Neurosci 12: 453-466.

66. De Filippo C, Cavalieri D, Di Paola M, Ramazzotti M, Poullet JB, et al. (2010) Impact of diet in shaping gut microbiota revealed by a comparative study in children from Europe and rural Africa. Proc Natl Acad Sci USA 107: 14691-14696.

67. Nguyen NT, Nakahama T, Le DH, Van Son L, Chu HH, et al. (2014) Aryl hydrocarbon receptor and kynurenine: Recent advances in autoimmune disease research. Front Immunol 5: 551.

68. Veldhoen M, Brucklacher-Waldert V (2012) Dietary influences on intestinal immunity. Nat Rev Immunol 12: 696708.

69. Benson JM, Shepherd DM (2011) Dietary ligands of the aryl hydrocarbon receptor induce anti-inflammatory and immunoregulatory effects on murine dendritic cells. Toxicol Sci 124: 327-338.

70. Bakdash G, Vogelpoel LTC, van Capel TMM, Kapsenberg ML, de Jong EC (2015) Retinoic acid primes human dendritic cells to induce gut-homing, IL-10-producing regulatory T cells. Mucosal Immunol 8: 265-278.

71. Lu L, Lan Q, Li Z, Zhou X, Gu J, et al. (2014) Critical role of all-trans retinoic acid in stabilizing human natural regulatory T cells under inflammatory conditions. Proc Natl Acad Sci USA 111: E3432-E3440.

72. Pizzorno JE, Murray M (2013) Text book of natural medicine. 4. St Louis: Churchill Livingstone Elsevier.

73. Melissa Vos, Giovanni Esposito, Janaka N Edirisinghe, Sven Vilain, Dominik M Haddad, et al. (2012) Vitamin K2 is a mitochondrial electron carrier that rescues pink1 deficiency. Science 336: 1306-1310.

74. Pickrell AM, Youle RJ (2015) The roles of PINK1, Parkin and Mitochondrial fidelity in Parkinson's Disease. Neuron 85: 257-273.

75. Moriya M, Nakatsuji Y, Okuno T, Hamasaki T, Sawada M, et al. (2005) Vitamin K2 ameliorates experimental autoimmune encephalomyelitis in Lewis rats. J Neuroimmunol 170: $11-20$

76. Shearer MJ, Newman P (2008) Metabolism and cell biology of vitamin K. Thromb Haemost 100: 530-547. 